\title{
A study of within-subject reliability of the brain's default-mode network
}

\author{
Merel Charlotte Postema ${ }^{1,2,3} \cdot$ Matteo De Marco $^{1}$ (1) $\cdot$ Elisa Colato ${ }^{1} \cdot$ Annalena Venneri $^{1}$
}

Received: 7 May 2018 / Revised: 19 December 2018 / Accepted: 21 December 2018 / Published online: 7 February 2019

(c) The Author(s) 2019

\begin{abstract}
Objective Resting-state functional magnetic resonance imaging (fMRI) is promising for Alzheimer's disease (AD). This study aimed to examine short-term reliability of the default-mode network (DMN), one of the main haemodynamic patterns of the brain.

Materials and methods Using a 1.5 T Philips Achieva scanner, two consecutive resting-state fMRI runs were acquired on 69 healthy adults, 62 patients with mild cognitive impairment (MCI) due to $\mathrm{AD}$, and 28 patients with $\mathrm{AD}$ dementia. The anterior and posterior DMN and, as control, the visual-processing network (VPN) were computed using two different methodologies: connectivity of predetermined seeds (theory-driven) and dual regression (data-driven). Divergence and convergence in network strength and topography were calculated with paired $t$ tests, global correlation coefficients, voxel-based correlation maps, and indices of reliability.

Results No topographical differences were found in any of the networks. High correlations and reliability were found in the posterior DMN of healthy adults and MCI patients. Lower reliability was found in the anterior DMN and in the VPN, and in the posterior DMN of dementia patients.

Discussion Strength and topography of the posterior DMN appear relatively stable and reliable over a short-term period of acquisition but with some degree of variability across clinical samples.
\end{abstract}

Keywords Brain imaging $\cdot \mathrm{fMRI} \cdot$ Hemodynamics $\cdot$ Reproducibility of results

\section{Introduction}

Resting-state functional magnetic resonance imaging (fMRI) holds great potential for clinical application due to its high level of availability, relatively high spatial resolution $(2-4 \mathrm{~mm})$, and its non-invasive manner to capture brain function [1-4]. In addition, it does not require cognitive task performance and there is evidence that it can contribute significantly to high levels of individual classification accuracy

Matteo De Marco

m.demarco@sheffield.ac.uk

1 Department of Neuroscience, University of Sheffield, Royal Hallamshire Hospital, Beech Hill Road, N Floor, Room N133, Sheffield S10 2RX, UK

2 Faculty of Earth and Life Sciences, VU University Amsterdam, Amsterdam, The Netherlands

3 Present Address: Language and Genetics Department, Max Planck Institute for Psycholinguistics, Nijmegen, The Netherlands in prodromal neurodegeneration due to Alzheimer's disease (AD) [5-7].

The analytical procedure of resting-state fMRI is centred on low-frequency $(<0.1 \mathrm{~Hz})$ fluctuations in the Blood Oxygen Level-Dependent (BOLD) signal $[8,9]$ that are dependent on the paramagnetic properties of deoxygenated haemoglobin [10]. Enhanced excitatory neuronal activity brings about an increase in oxygenated blood supply and a subsequent decrease in deoxygenated haemoglobin levels $[11,12]$. This leads to decreased dephasing of excited spins and a subsequent increase in $\mathrm{T} 2 *$ decay time, reflected by an increase in BOLD-signal intensity [13].

Even during "rest" (i.e., the absence of a specific cognitive task), the brain maintains high levels of neuronal activity [14]. This is coherently organised into networks based on patterns of spontaneous synchronised activity in different brain areas [2]. This synchronous activity is referred to as functional connectivity, and studies on neurodegenerative conditions suggest that the patterns of connectivity reflect the underlying neural anatomy [15]. Various resting-state 
networks have been identified, including the visual-processing network (VPN), the sensorimotor network, the frontoparietal control network, the salience network, and the default-mode network (DMN) [16, 17]. The DMN is unique in showing a robust decrease in activity during cognitive task performance [14, 17], which is essential for successful engagement in overt cognitive processing, e.g., memory encoding [18]. Activation of the DMN also directly sustains cognitive processes, including autobiographical episodic memory retrieval $[16,19,20]$, self-referential mental processing [16, 19], and mind wandering [21]. The topography of the DMN encompasses several brain areas and often an anterior and a posterior component are distinguished. Limbic areas are also involved in this network including the posterior cingulate cortex, retrosplenial cortex, hippocampus, and precuneus [22-24]. The anterior DMN centres in the medial prefrontal cortex and ventral anterior cingulate cortex [23, 25], while the posterior DMN centres in the inferior parietal lobule and several temporal lobe areas. Anterior and posterior DMNs also show distinct alterations in functional activity that appears to decrease with age in the posterior DMN, and both decreases and increases with age in the anterior DMN [26]. Such an increase in activity is believed to serve as a compensatory mechanism to optimise levels of cognitive performance along the axis of age-related neurological changes [27].

Interestingly, alterations in DMN activity, different from the ones observed in normal ageing [28], have been reported in several brain disorders, such as Alzheimer's disease (AD) $[15,19] . \mathrm{AD}$ is a progressive neurodegenerative disorder characterised, at the pathological level, by aberrant accumulation of amyloid-beta and tau proteins into extracellular amyloid plaques and intracellular neurofibrillary tangles, respectively [29]. Since initial amyloid deposition has been observed in several regions of the DMN, including posterior cingulate cortex, retrosplenial cortex, and lateral parietal cortex [30], and because diminished DMN functional connectivity has been observed in mild AD patients and patients at the mild cognitive impairment (MCI) prodromal stage of $\mathrm{AD}$ (see [16] for a review), measures of DMN functional activity with resting-state fMRI promise to become a possible biomarker of early AD [22, 31].

For resting-state fMRI to be successfully applied clinically, however, reliability of the acquired signal in the context of neurodegeneration needs to be established. Although several test-retest reliability studies of resting-state fMRI have been carried out in young healthy adults [1, 32-39], few studies have focused on healthy elderly [40] and MCI patients [41], and, to our knowledge, no such studies have been carried out in AD patients. Usually, the study of reliability of brain networks requires longitudinal designs and repeated follow-ups that are not always possible in clinical populations, especially those with a neurodegenerative condition characterised by progressive neural loss/dysfunction such as AD. The aim of this study, therefore, was to investigate the reliability of signal acquired with resting-state fMRI in the DMN in healthy elderly adults, MCI patients, and $\mathrm{AD}$ patients in the short term, following a procedure which exploits the processing of multiple runs acquired within the same fMRI session. All participants were scanned once, but across two different runs. Data were analysed using two different methodologies: seed-based linear functional connectivity and dual-regression analysis [42]. These are major methodologies widely implemented for the analysis of brain networks. Concurrent analytical approaches were chosen to maximise conservativeness and test the presence of both differences and similarities. Differences between Run 1 and Run 2 were tested by means of paired $t$ tests run to compare the spatial map of each network calculated on each run. Run 1 is usually an acquisition during which the participants acclimatise to the scanner environment, whereas Run 2 may be associated with more fatigue, decreased wakefulness, and more movements. For this reason, the null hypothesis was accompanied by an alternative hypothesis testing whether significant differences in the DMN would exist between the two runs.

Voxel-based correlation between the two runs was instead calculated to test the similarities of the two sets of maps. In addition, analysis of network strength at the major hubs within each map was carried out from seed-based networks. For this purpose, further numerical indices of functional connectivity were extracted with a seed-based approach, using an anatomical atlas. Multiple measures of statistical correlation and consistency were calculated: Pearson's correlation, Cronbach's alpha, and intraclass correlation.

\section{Materials and methods}

\section{Participants and data acquisition}

Seventy-two healthy volunteers, 67 patients with MCI, and 31 patients with dementia of the $\mathrm{AD}$ type were recruited at the IRCCS Fondazione Ospedale San Camillo, Venice, Italy, as part of a wider project studying cognitive efficiency in physiological and pathological ageing (Grant no 42/RF-2010-2321718 by the Italian Ministry of Health to AV) and their data contributed to the Venice Lido Ageing Database. This study had received approval by the Institutional Review Board of the IRCCS Fondazione Ospedale San Camillo (Venice, Italy), (Protocol N. 11/09 version 2). Informed consent was obtained from all individual participants included in the study. Diagnostic status was established based on a consensus among clinicians. All participants, aged $>45$ years, underwent complete neurological screening to rule out the presence of clinical exclusion 
criteria (extensively described in De Marco et al. [43]). To determine the extent of cognitive impairment, a comprehensive neuropsychological-test battery was used (see [5]), and scores derived from this battery were used to reach a consensus diagnosis.

T2*-weighted brain MRI sequences were acquired with a 1.5 T Philips Achieva MRI scanner. To enable assessment of the set exclusion criteria, T1-weighted, T2-weighted, and FLAIR scans were also obtained. Before scanning, participants were instructed to close their eyes and remain still for the whole duration of the session, but to remain awake. Resting-state fMRI scans were not preceded by any specific cognitive tasks.

The $\mathrm{T} 2 *$-weighted images were acquired using the following scanning parameters: $\mathrm{TR}=2 \mathrm{~s}$, echo delay time $=50 \mathrm{~ms}$, flip angle $90^{\circ}$, voxel dimensions $2.875 \times 2.875 \times 6 \mathrm{~mm}$, matrix size $80 \times 80 \times 20$, and field of view $230 \mathrm{~mm}$. Two 120 -volume runs of 20 contiguous axial slices obtained in ascending order were acquired for each participant. Twenty seconds of dummy scans were acquired before each run to enable longitudinal magnetisation to reach equilibrium.

A complete scanning session took approximately $35 \mathrm{~min}$, during which several images were acquired. In this study, only $\mathrm{T} 2{ }^{*}$-weighted images were used for analysis. A T $2 *_{-}$ scanning session took approximately $10 \mathrm{~min}$, during which two consecutive runs were acquired, each lasting $4 \mathrm{~min}$ and $20 \mathrm{~s}$, including $20 \mathrm{~s}$ during which 10 dummy volumes were acquired.

\section{fMRI data pre-processing}

Data were pre-processed and analysed using Statistical Parametric Mapping (SPM) 8 software (Wellcome Centre for Human Neuroimaging, London, UK) implemented in MATLAB R2014a (Mathworks Inc., UK). All echo planar scans were corrected for slice timing [44], and each of the two volume runs was independently realigned [45]. This option allows the creation of mean volumes as reference and the estimation of six linear and rotational rigid body motion parameters that were visually inspected to identify problematic head movements. Participants with a translational and/or rotational movement that exceeded $1.5 \mathrm{~mm}$ or $3^{\circ}$, respectively, were excluded from the analysis. These included two healthy participants, five MCI patients, and two $\mathrm{AD}$ dementia patients. This is a procedure that is widely used to minimise the impact of excessive motion [46-50]. One additional patient with dementia and one additional control were excluded because of signal artefacts. The final data set included 69 healthy participants ( 27 males; mean age $=66$ years \pm 8.40 ; mean MMSE-score $=29.09$; mean education level $=11.41$ years), $62 \mathrm{MCI}$ patients ( 30 males; mean age $=74$ years \pm 6.22 ; mean MMSE-score $=27.73$; mean education level $=10.76$ years) and 28 patients with
AD dementia ( 14 males; mean age $=75$ years \pm 7.50 ; mean MMSE-score $=21.18$; mean education level $=8.11$ years) . Between-group matching for demographic characteristics was not necessary, because no between-group comparison was carried out.

After realignment, scans were corrected for individual brain differences using spatial normalisation, which includes co-registration of the fMRI time series with the standard SPM echo planar imaging (EPI) template, and volumebased registration to normalise the EPI data to the Montreal Neurological Institute (MNI) template with known standard space [51]. Spatial normalisation was carried out for both runs separately, using the first realigned volume of the first run and the first realigned volume of the second run as source images to match the EPI template. The normalised voxel size was set at $2.00 \times 2.00 \times 2.00 \mathrm{~mm}$. Next, a bandpass filter between 0.008 and $0.100 \mathrm{~Hz}$ [14] was applied using the REST toolbox (http://www.restfmri.net) in SPM 8 to remove part of non-neural sources of variability in the BOLD signal (mostly due to cardiorespiratory factors and to slow signal drift). With specific focus on cardiorespiratory rhythms, since these are pseudoperiodic, it was assumed that both cardiac and respiratory signals would be regressed out when analysed with within-subject designs in a way similar to that routinely implemented for modelling of task-based fMRI. Finally, signal-to-noise ratio was improved by spatially smoothing the fMRI images using an isotropic Gaussian kernel of $6 \mathrm{~mm}^{3}$ full-width at half-maximum [52].

\section{Cognitive and neurostructural characterisation of the cohort}

Scores obtained on representative neuropsychological tests of particular relevance in ageing and neurodegeneration of the AD type were extracted and confronted between groups. As shown in Table 1, significant clinical differences were visible across the three diagnostic groups for all measures.

The three diagnostic groups were further characterised by analysing the global volumetric properties of their brains. The T1-weighted images were segmented following the standard SPM routine, and native-space maps of grey matter, white matter and cerebrospinal fluid were quantified in millilitres using the "get_totals" command line (http://www. cs.ucl.ac.uk/staff/g.ridgway/vbm/get_totals.m). Total intracranial volumes were then computed summing up the volume of the three tissue classes, and the fraction of grey matter (an index of global brain atrophy) was then obtained by fractionating grey matter volume by total intracranial volume. Three independent-sample $t$ tests were then run to compare the levels of global atrophy among the three groups. These resulted to be all significant (all $p$ values $<0.05$ ), indicating that the three groups had different levels of cerebral atrophy due to neurodegenerative processes. Based on this, it was decided 
Table 1 Cognitive characteristics of the cohort. Mean (and standard deviation) is shown for each test

\begin{tabular}{llcl}
\hline & Healthy adults $(n=69)$ & MCI $(n=62)$ & $\begin{array}{l}\text { AD demen- } \\
\text { tia patients } \\
(n=28)\end{array}$ \\
\hline Mini mental state examination & $29.09(1.21)$ & $27.73(4.22)$ & $21.18(2.54)$ \\
Category fluency test & $42.80(9.42)$ & $29.34(9.84)$ & $16.46(5.84)$ \\
WAIS-Similarities & $21.30(4.74)$ & $19.08(4.14)$ & $10.52(4.28)$ \\
Prose memory test—delayed recall & $13.00(4.61)$ & $7.27(4.58)$ & $1.93(2.81)$ \\
Rey complex figure-delayed recall & $16.33(5.55)$ & $9.68(13.41)$ & $2.62(4.03)$ \\
\hline
\end{tabular}

Clinical instruments measuring general levels of cognition, episodic memory, and semantic processing are included. All group difference were significant at a Bonferroni-corrected $p<0.05$ not to pool all participants in a single group, but apply study procedures in a separate way for each diagnostic group.

\section{Approaches to fMRI processing}

To address the experimental question from multiple angles, the two runs were investigated in terms of divergence and convergence (i.e., testing both differences and similarities between runs). The analysis of divergence/differences was devised mainly to rule out the presence of major discrepancies between the two runs and served as a prerequisite to the main analyses.

Two complementary analytical routes were defined for the calculation of individual maps of network functional connectivity and for testing similarities and differences between runs. A seed-based methodology was implemented as a theory-driven approach, and a dual regression was implemented as a data-driven approach. The a priori choice of seeds enabled us to focus on these regions more in detail. Conversely, the data-driven definition of maps allowed us to test similarities between runs in a voxel-by-voxel way.

\section{Theory-driven approach: seed-based functional connectivity networks}

A predetermined set of seed regions was selected based on the Automatic Atlas Labelling [53], and were constructed using the WFU PickAtlas toolbox [54]. The seeds included the posterior cingulate cortex and the medial prefrontal cortex for the calculation of the posterior and anterior DMN, respectively, and the calcarine cortex for the calculation of the VPN as methodological control (Fig. 1). Additional seeds were drawn in the white matter and in the cerebrospinal fluid. Seed-based timecourses were extracted from each seed region using the MarsBAR toolbox [55]. Individual maps of functional connectivity were computed modelling the linear association between the timecourse of the seed and the timecourse of each cerebral voxel. The signal extracted from the map of white matter and cerebrospinal fluid was regressed out, together with the six linear and rotational rigid body motion parameters, their squared values, their temporal difference, and the square of the differenced values, for a total of 24 regressors [56]. These regressors were calculated using the $m p \_$diffpow 24 script (https://www.warwi ck.ac.uk/fac/sci/statistics/staff/academic-research/nichols/ scripts/spm/mp_diffpow24.sh).

A fervent debate is still ongoing on as to whether wholebrain signal should also be regressed out from individual linear models [57]. In this study, this was not carried out, as it might lead to spurious negative correlations within individual models, which, in turn, lead to altered group-level connectivity patterns [58].

\section{Data-driven approach: dual-regression networks}

A second, multiple-step approach was also followed: a dualregression procedure was carried out $[42,59]$ using a series of MATLAB and SPM routines. Briefly, this technique processes the spatial outline of a set of maps generated with an independent component analysis, a technique that decomposes the entire fMRI data set into a selected number of latent variables (components), each of which corresponds to an independent source of signal and has its own specific topography $[6,16]$. Components of interest (corresponding to the topography of brain networks of interest) are identified, and an average individual timecourse within the contour of the component is extracted. Maps of connectivity are then calculated by modelling the linear association between this global timecourse and that of each single voxel. This was carried out regressing out the same nuisance vectors as per seed-based networks (Sect. 2.4.1). Given the datadriven nature of this approach and the profound neurological differences between healthy adults and patients, three separate independent component analyses were run, one per each diagnostic group. The fMRI toolbox GIFT (GIFT, v1.3i, http://www.mialab.mrn.org/software/gift) was used, in combination with the Infomax optimisation principle and the number of components to be extracted was set at 20 , as landmark research has proficiently used this number of components to identify the major haemodynamic networks [60]. Among the 20 components, the anterior and posterior 
Fig. 1 Three ROIs devised as part of the theory-driven section of the study methodology. These were constructed based on an anatomical atlas as main seed of the anterior DMN (medial prefrontal cortex, red), posterior DMN (posterior cingulate cortex, blue), and VPN (calcarine cortex, green)

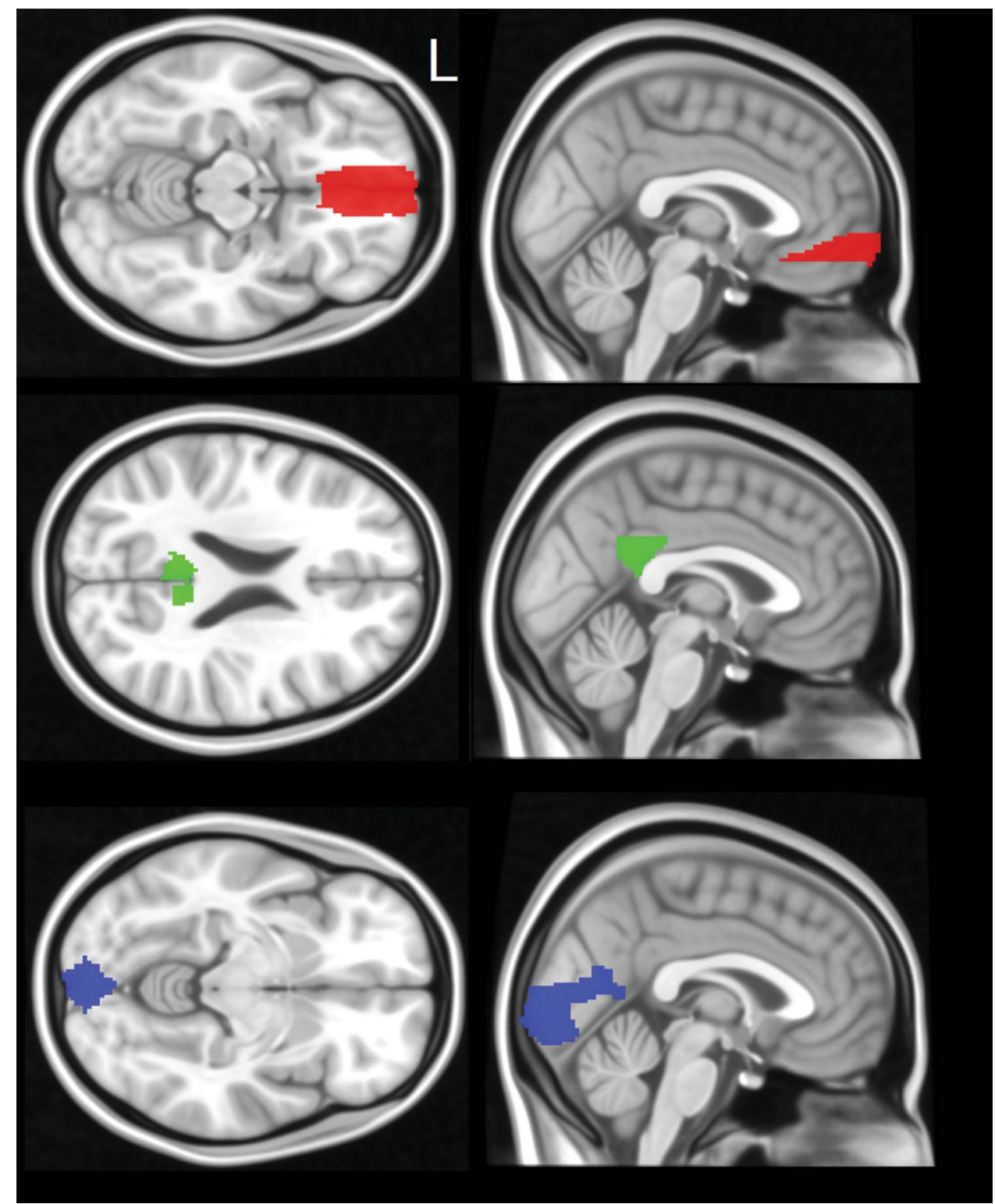

DMNs and VPN were identified by three independent raters, who obtained $100 \%$ agreement on the final selection [61].

\section{Inferential models}

\section{Investigating divergence}

Paired $t$ tests were carried out in SPM per group to assess individual differences in seed-based and dual-regression network topography between runs, using significance thresholds of $p=0.0005$ (uncorrected) at the set level, and $p=0.05$ (false discovery rate corrected) at the cluster level, as reported by the SPM output. False discovery rate was preferred over familywise error correction, because it defines a more liberal threshold for rejecting the null hypothesis [62]. As far as the specifications of this study are concerned, however, (i.e., finding confirmatory evidence in support of similarity between runs), this translated into the more conservative choice. According to the alternative hypothesis, significant differences would exist given that participants tend to experience fatigue, decreased wakefulness, and more movements during Run 2.

\section{Investigating convergence}

Initially, the similarity between the two maps was qualitatively inspected by running a conjunction analysis between maps generated from Run 1 and Run 2. This was done for all maps of connectivity.

Second (and in specific compliance with the remit of the hypothesis), correlational models were devised to analyse statistical similarities between Run 1 and Run 2 . 
These were implemented as voxel-based whole-brain correlations as part of the data-driven approach (processing, therefore, maps of connectivity created after dual regression), and as regional correlations limited to the ROIs as part of the theory-driven approach (thus processing maps of connectivity obtained with seed-based models.

Theory-driven approach: consistency of ROIs' statistics Following the definition of seed-based maps, local indices of the three seeds (described in Sect. 2.4.1 and illustrated in Fig. 1) were further analysed. Data were extracted from these three ROIs for each participant individually and exported to MATLAB. IBM SPSS Statistics 23 was further used for statistical analysis. To examine if Run 1 and Run 2 were correlated with one another, a bivariate Pearson's correlation model was initially carried out. As a short-term within-subject design was used, there was no need to correct for factors like age and $\mathrm{AD}$ pathology. Since Pearson's product-moment solely examines a relationship between runs and does not indicate internal consistency, other indices of reliability were computed, namely, intraclass correlation and Cronbach's alpha [63, 64]. Indices of reliability were run in the entire cohort and within each diagnostic group. Intraclass correlation was set to capture absolute agreement reliability in the numerical estimate between runs.

Data-driven approach: global and voxel-based whole-brain correlations Both global and regional properties of individual maps of functional connectivity obtained from the dual regression were analysed. First, individual maps of connectivity were reshaped into unidimensional data matrices for the calculation of coefficients of correlation across the entire network map of each participant. These were then plotted at a group level to characterise the global strength of correlation coefficients. Second, voxel-by-voxel correlations were tested with the Biological Parameter Mapping toolbox [65], fully implemented in SPM. This toolbox allows the calculation of whole-brain maps of positive and negative correlations between two sets of inter-dependent maps. The presence of a positive coefficient of correlation between Run 1 and Run 2 was tested for each voxel. A statistical threshold of $r>0.6$ was applied to focus exclusively on the strongest associations. The resulting maps were inspected and interpreted in the light of the expected map of the outcome (i.e., the outline of the network).

\section{Results}

No significant differences emerged from any of the $t$ test models. Moreover, all networks of healthy elderly, MCI patients, and AD patients showed high topographical similarity between runs. This is illustrated in Figs. 2 and 3, which show the results of the one-sample $t$ tests and the conjunction analyses for the maps emerged from seedbased and dual-regression procedures.

Regional ROI indices of connectivity emerged from seed-based models were plotted and inspected to verify normality across the three diagnostic groups. To do so, each distribution was visually compared to that of multiple vectors randomly extracted from a normal distribution having the same mean and standard deviation as the measured data (this was carried out using $R$ environment and the "rnorm" function). In no case did the distribution of peak scores suggest breach of normality.

Bivariate correlation analyses (illustrated in Fig. 4) revealed that the relationship between runs was very solid in the posterior DMN ( $p$ values $<0.001$ ). Overall, the anterior DMN and the VPN yielded weaker coefficients of correlation. Cronbach's alpha values and intraclass correlation coefficients are included in Table 2. Overall, the results mimicked the outcome of the coefficient models, with Cronbach's alpha and intraclass correlation coefficient converging towards an average $>0.8$ reliability for the pDMN in each diagnostic group. The reliability of both aDMN and VPN was associated with variable but overall smaller indices.

Individual indices of Run 1-Run 2 correlation across the entire reshaped network map are illustrated in Fig. 5 and show global robust similarity between the two runs ( $r$ scores tended to distribute near 0.5 ). The voxel-by-voxel correlation between the maps of functional connectivity calculated with dual regression on Run 1 and Run 2 is shown in Fig. 6. The results are aligned with the outcome of the ROI-based analyses: no well-defined patterns were found in association with the anterior DMN and VPN (although a trend emerged within the occipital pole for the latter network). Solid correlations within the posteromedial and inferior parietal hubs of the posterior DMN were found instead across all three diagnostic groups, interestingly with a gradual decline observed in patients with MCI and AD dementia. To test whether differences in motion between runs were the source of decreased reliability in patients, we computed for each run an index of absolute framewise displacement [66]. Briefly, this index is a measure of the average linear and rotational displacement shown over the entire run. Paired $t$ test comparisons were run to compare the two runs separately for each diagnostic status. No significant differences were found. 


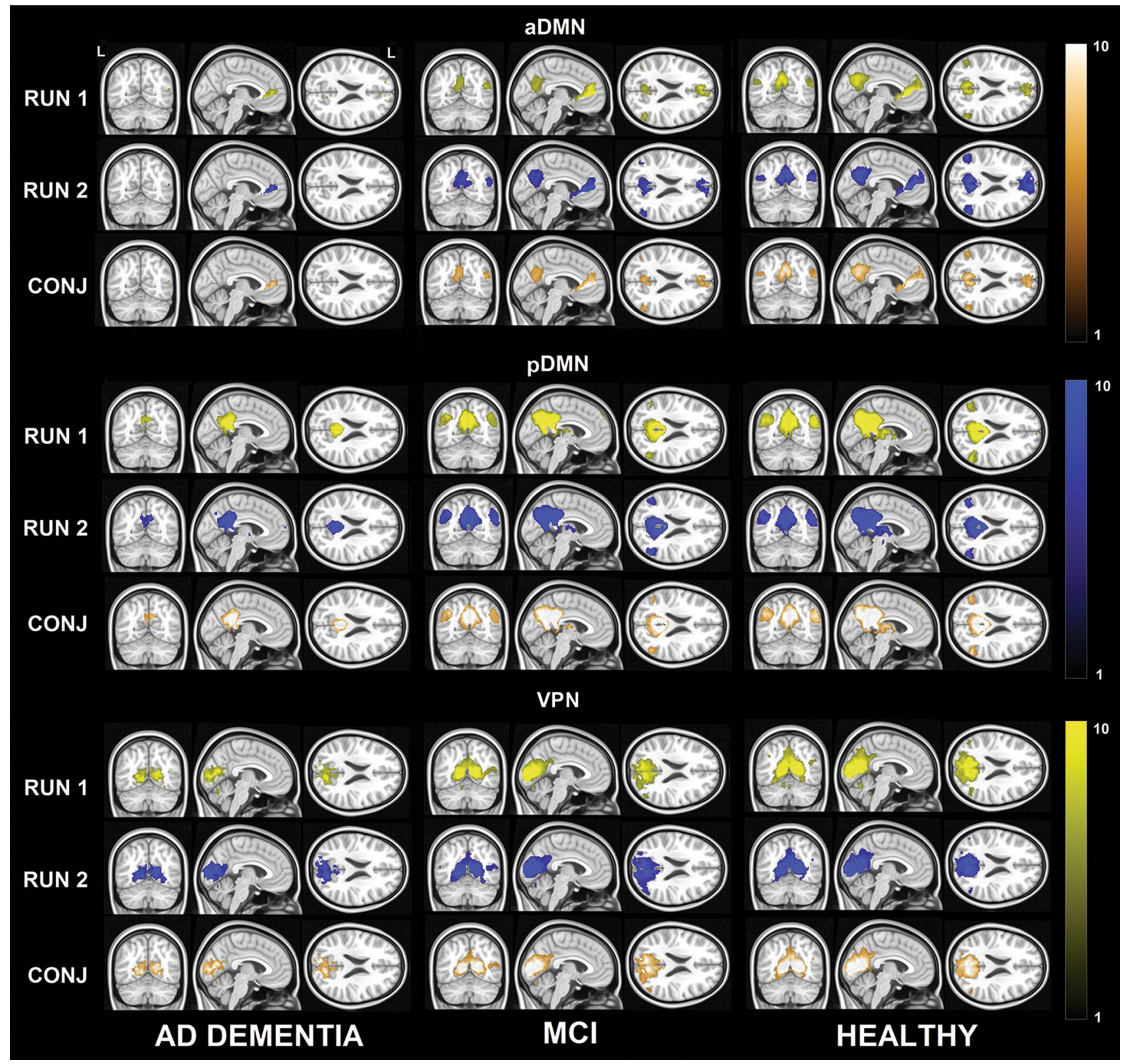

Fig. 2 Maps of the anterior and posterior DMN and the VPN, as calculated from seed-based models via one-sample $t$ tests. The strength of the haemodynamic connectivity which constitutes this network is illustrated separately for each diagnostic group: during Run 1 (yel-

\section{Discussion}

In this study, we investigated short-term reliability of resting-state fMRI, with a particular focus on the DMN, in healthy elderly, MCI patient, and AD patient samples. To do so, we ran both statistical models devised to detect differences between the two runs, and statistical models devised instead to capture the degree of similarity between the two runs. In fact, the absence of significant differences between runs emerged from paired-sample $t$ tests (and thus, low) and Run 2 (blue). The conjunction analysis is illustrated in gold MNI coordinates: $x=-4 ; y=-62 ; z=22$. Colours indicate the statistical strength of the $z$ statistics. A legend is included on the right-hand side, CONJ conjunction

the non-rejectability of the null hypothesis) is itself not sufficient to support the concept of analogy between runs. For this reason, paired comparisons were flanked by more conventional measures of statistical similarity. In addition, maps of network connectivity were computed using two different theory-driven and data-driven approaches: seed-based models and a dual-regression procedure, respectively.

At present, indices of functional connectivity are not recognised as validated biomarkers for AD. This study was carried out with this clinical objective in mind, in support 


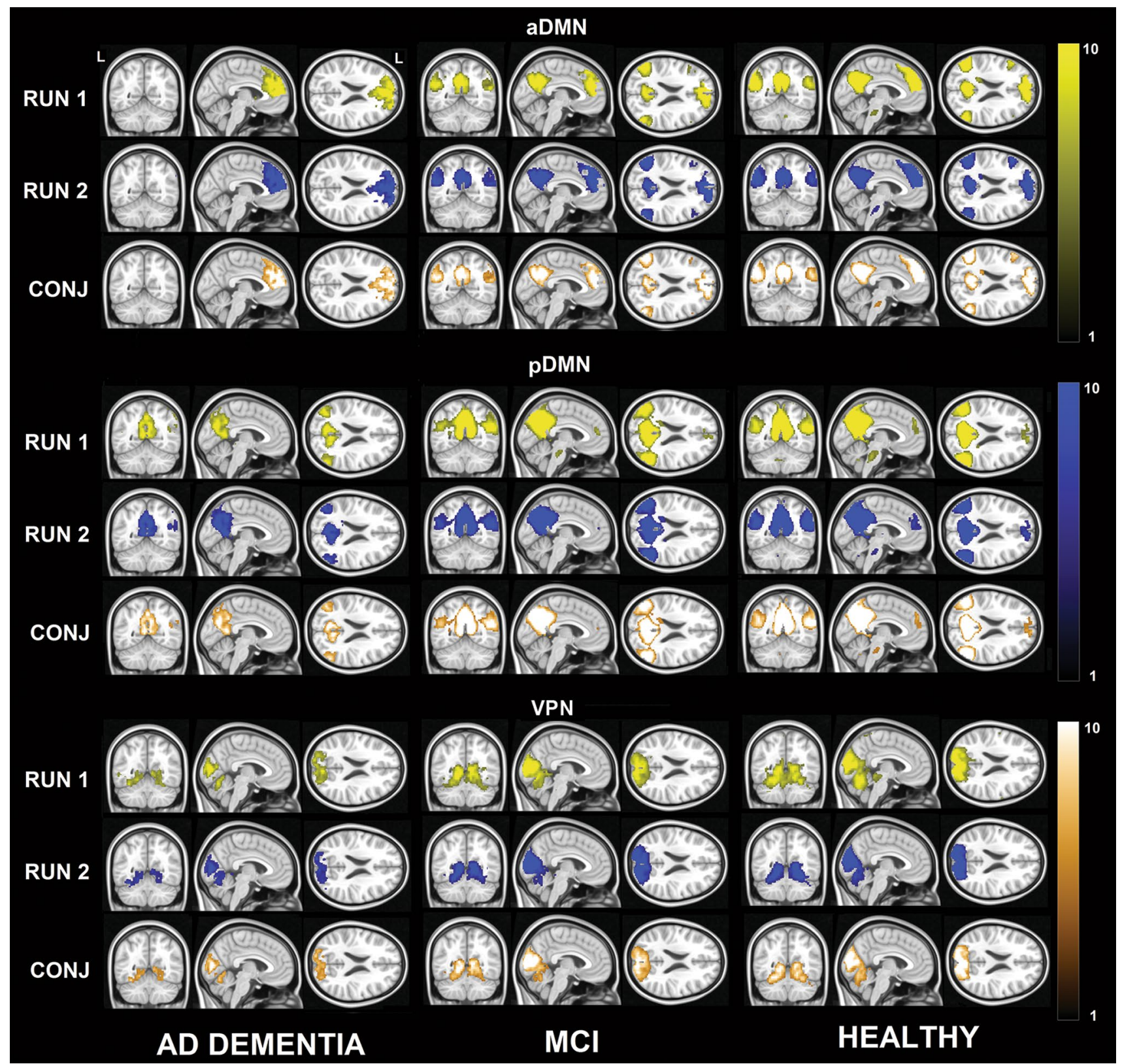

Fig. 3 Maps of the anterior and posterior DMN and the VPN, as calculated based on the dual-regression procedure via one-sample $t$ tests. The strength of the haemodynamic connectivity which constitutes this network is illustrated separately for each diagnostic group: during Run 1 (yellow) and Run 2 (blue). The conjunction analysis is illustrated in gold. MNI coordinates: $x=-4 ; y=-56 ; z=22$. Colours indicate the statistical strength of the $z$ statistics. A legend is included on the right-hand side. $C O N J$ conjunction of a potential future applicability of these measures as clinical biomarker proxies of abnormal ageing. Results indicated high within-subject reliability in the posterior DMN in healthy controls and patients, as confirmed by all statistical methods, including those testing the hypothesis of significant differences between runs, and those testing statistical similarities. The reliability of the pDMN in MCI and AD dementia, however, showed a declining trend, as confirmed in voxel-by-voxel models. Lower signal reliability was observed in the anterior DMN and in the VPN. This partially goes against our prediction of reliability measures becoming less efficient as disease progresses, because poor reliability was seen in all groups.

Since biomarker validity depends on both within- and between-subject variabilities [67], our results only partly support the applicability of the entire construct of restingstate fMRI as a potential indicator of abnormal ageing. The reason for focusing on within-subject reliability is to 

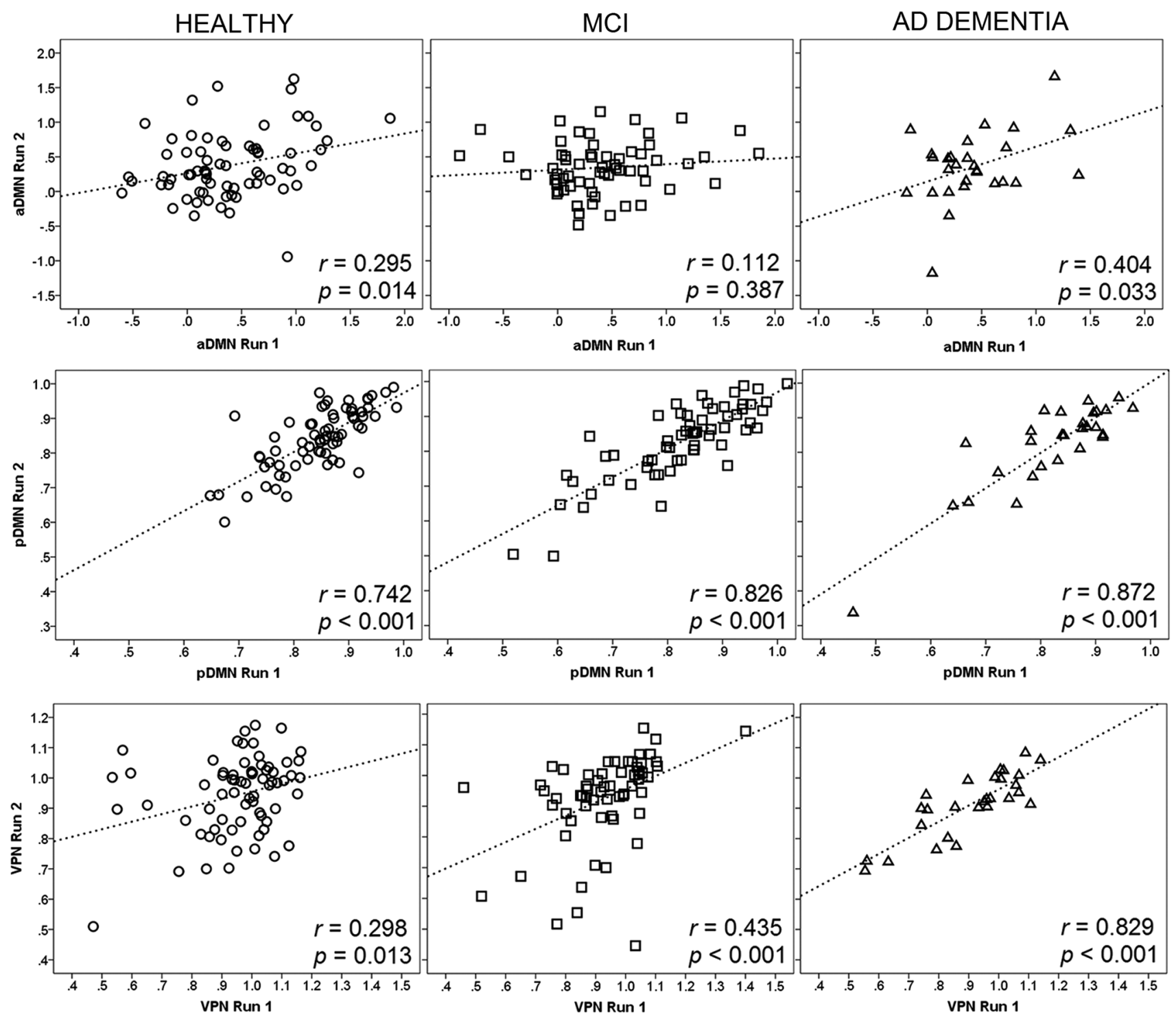

Fig. 4 Correlation graph of mean functional activity in the central ROIs of each network (shown in Fig. 1) as emerged from seed-based models: anterior default-mode network $(\mathrm{aDMN})$, posterior default-

assess reliability while leaving out sample heterogeneity, which consequently enables generalisation of results [68, 69]. For this reason, we expected Cronbach's alpha to be the key measure of reliability, rather than the intraclass correlation coefficient $[1,37,40,41]$. In this study, however, the results provided by these two measures were comparable. Even though the intraclass correlation coefficient has multiple subtypes that, when chosen incorrectly, can influence reliability estimation [70], we found almost identical results across all subtypes.

Signal stability of fMRI might also be influenced by various other factors. Although AD pathology could not have influenced our results-because we used a within-subject design and scans were acquired consecutively over the span mode network (pDMN), and visual-processing network (VPN). Pearson's correlation coefficients and corresponding $p$ values are also included

of a few minutes-factors such as machine noise, experimental instructions, data analysis strategy, and physiological noise could still have affected reliability [67]. In this study, only the latter factor could have influenced signal acquisition and could account for the observed group differences, as all participants were scanned using the same scanner, given the same instructions and analysed following the same data analysis strategy. Physiological noise encompasses cardiac-, respiratory-, and head motion and is known to increase with increasing signal-to-noise ratio [71, 72]. As we used a $1.5 \mathrm{~T}$ system in this study, the influence of cardiac- and respiratory noise should be relatively low, and should be minimised by the use of a within-subject design. It is likely, however, that head motion might have in part 
Table 2 Indices of reliability computed from seed-based maps of network connectivity

\begin{tabular}{lllll}
\hline Reliability index & Entire cohort & Healthy & MCI & AD dementia \\
\hline Anterior DMN & & & & \\
Cronbach's alpha & 0.392 & 0.456 & 0.192 & 0.565 \\
$\begin{array}{l}\text { Intraclass correla- } \\
\text { tion }\end{array}$ & 0.392 & 0.459 & 0.193 & 0.569 \\
$\begin{array}{l}\text { Posterior DMN } \\
\text { Cronbach's alpha }\end{array}$ & 0.895 & 0.905 & 0.847 & 0.925 \\
$\begin{array}{l}\text { Intraclass correla- } \\
\text { tion }\end{array}$ & 0.895 & 0.906 & 0.848 & 0.928 \\
$\begin{array}{l}\text { VPN } \\
\text { Cronbach's alpha }\end{array}$ & 0.602 & 0.453 & 0.859 & 0.606 \\
$\quad \begin{array}{l}\text { Intraclass correla- } \\
\text { tion }\end{array}$ & 0.604 & 0.456 & 0.863 & 0.610 \\
\hline
\end{tabular}

affected our data. Despite correcting for volume misalignment by means of spatial realignment, head motion is known to induce additional secondary biases, such as spin-history effects and magnetic field inhomogeneities [73]. Additional strategies have been proposed to correct for motion artefacts, aside from that implemented in this work [56, 74]. A number of these novel techniques, however, have been noted to disrupt or alter the autocorrelation structure of fMRI time series [75]. Since in-scanner motion is a major source of false positives, we are open to the possibility that the use of other methodologies may lead to slightly different findings. It is fair to acknowledge, however, that the results of the paired $t$ tests were negative (i.e., no difference between runs) and that voxel-based correlation of the $\mathrm{pDMN}$ was limited to the network contour, and thus, false positives do not represent an issue in this study.

Signal fluctuations could have been additionally influenced by differences in eyes open and closed states, which may have resulted in significant signal differences in the
VPN. Although all participants were instructed to keep their eyes closed during the whole duration of the scanning session, it is possible that some of them might have opened their eyes. McAvoy and colleagues, for instance, reported increased BOLD-signal intensity in the VPN, sensorimotor, auditory network, and retrosplenial cortex during eyes closed compared to eyes open resting states [76]. Since no camera was available in the scanner to monitor eye closure, the possible confounding influence of eye closure status on the BOLD signal cannot be ruled out. Moreover, although the definition of the DMN pattern (or that of the other networks) is easily reproducible across subjects [77], it is possible that different methodological choices (i.e., in the selection of the ICA optimisation principle or number of components, or in the use of a comparable yet different seed, e.g., the entire posterior cingulate cortex rather than the sole retrosplenial portion) may lead to slightly different results.

In addition, neuronal factors such as cognition and behaviour could have influenced within-subject signal fluctuation, as resting state is associated with unconstrained thoughts and subsequent unpredictable behaviour [78]. Nevertheless, it is unlikely that spontaneous behaviour constitutes the main source of resting-state fMRI BOLD-signal variance in this study, since robustness and spatial coherence of this type of signal have been reported in non-human primates and across various behavioural states (see [67] for a review). In addition, we have demonstrated particularly high within-subject reliability in higher order brain networks, consistent with other resting-state fMRI reliability studies (see [67] for a review).

In addition, false-positive findings and low power could have affected reliability. A recent article by Eklund and colleagues claimed that the parametric approaches used in SPM and other programmes used to analyse fMRI data, might generate up to $70 \%$ of false-positive findings, which is much larger than the generally accepted 5\% [79]. Their
Fig. 5 Group distribution of individual coefficients of correlation calculated over the entire network maps. Boxplots indicate medians and interquartile ranges
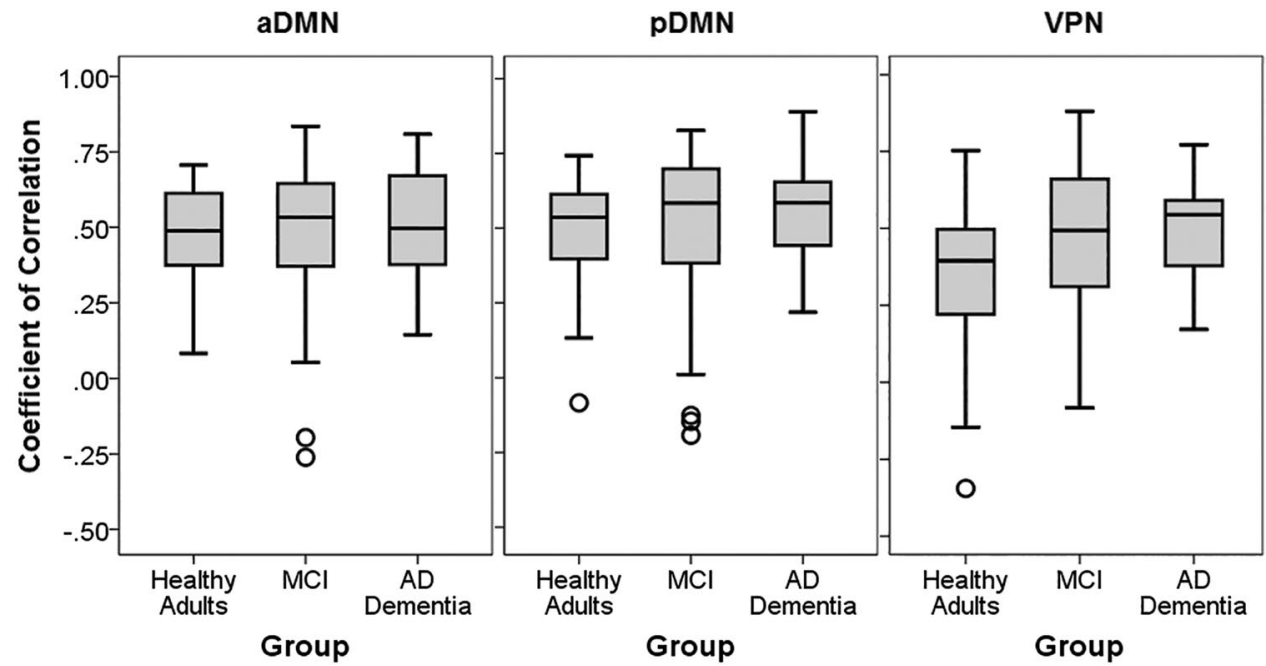
Fig. 6 Voxel-based correlational maps between Run 1 and Run 2 for each brain network computed with dual regression. Maps were thresholded at $r>0.6$

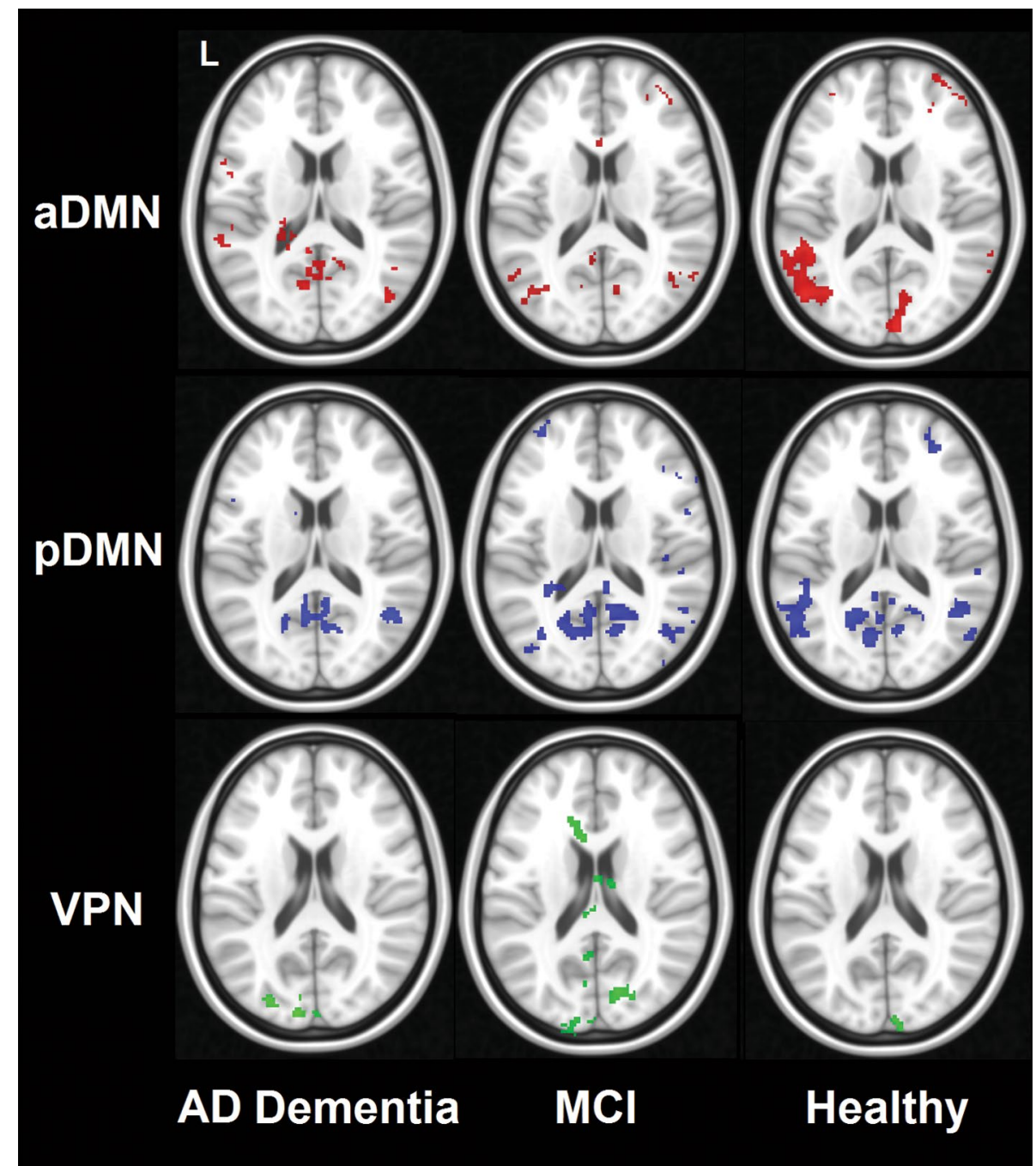

results might have immense consequences for earlier performed fMRI-based studies, yet they strengthen the non-significant findings in our study. Furthermore, sufficient power is needed to reject the null hypothesis. Since the sample size of AD patients $(n=28)$ was more than $50 \%$ smaller than that of MCI patients $(n=62)$ and healthy elderly adults $(n=69)$, it is possible that the relatively low power in the AD group increased the likelihood of non-significant findings in this sample [80]. Zandbelt and colleagues, however, have reported that a sample size of 30 would be adequate for sufficient power in studies of this kind [69].

Of the above-mentioned factors, head motion and eyes open/closed status are the most likely factors which might have affected our data, influencing aDMN and VPN at the whole-brain level. At the single-voxel level, however, signal fluctuation appeared to be relatively lower in AD patients. We tested the possibility that this decrease might have been spuriously due to larger between-run motion differences seen in patients as opposed to controls. The analysis of between-run differences in absolute displacement was convincing evidence to rule out this possibility.

It would be interesting to investigate the mechanisms underlying low signal reliability. Since the VPN is among the latest resting-state networks affected by amyloid pathology [81], yet it shows low levels of reliability, we suggest that signal reliability is not dependent on amyloid pathology per se, but that other mechanisms, which are associated with abnormal ageing, may affect its strength. This is also supported by the robust reliability found in the posterior DMN, which includes regions that are severely affected by amyloid pathology.

Thus, further research into the molecular mechanisms underlying decreased resting-state fMRI signal reliability is needed to increase insights into abnormal ageing. To obtain a complete overview of its applicability as an indicator of abnormal ageing, studies that examine between-subject variability are needed. Furthermore, future studies could examine signal reliability using different fMRI scanners with various magnetic field strengths to increase generalisation 
of these results further. In addition, long-term signal reliability studies should be carried out in healthy ageing to increase insights into the effect of treatment and/or disease progression on brain functional connectivity in MCI and $\mathrm{AD}$ patients. To examine the value of resting-state fMRI as a biomarker proxy at the individual level, one could further study signal fluctuation in single subjects, e.g., via correlational models. Voxel-by-voxel correlational maps, however, can only be calculated at a group level, not an individual level. In fact, fMRI data modelling at the individual level results in the computation of a three-dimensional map (one sole number per voxel). In addition, individual voxel-byvoxel correlations cannot be calculated along the axis of time either, since no overt association exists between the sequence of volumes of the first acquisition and the sequence of volumes of the subsequent acquisition. Consistently with these observations, in this study, we only carried out voxelwise models testing differences and similarities between runs at a group level only.

A methodological alternative to resting state is the study of task deactivations. Since the regions that de-activate during an overt task are the same that are active at rest, reliability in the pattern of deactivation may represent a surrogate methodology for the analysis of DMN network reliability. Deactivations, however, depend on the type and computational load of the task used as part of the experimental manipulation [82], and this might lead to BOLD differences that are partly due to practice effects, or inter-individual neural reserve [83]. Furthermore, no other networks could be studied with task deactivations.

Our results support the use of haemodynamic indices, computed from resting-state fMRI, as variables of interest for a future clinical translation into a potential biomarker proxy of incipient $\mathrm{AD}$ at the group level and demonstrate that resting-state fMRI could even be reliably applied to AD patients in short-term studies when a voxel-by-voxel approach is adopted. This would be particularly true for the posterior DMN. Certainly, classification studies with a specific focus on cross-cohort validation would provide a definite answer as to whether a DMN index might be effectively used in clinical routine. In addition, these findings may lead to a better understanding of disease mechanisms, i.e., short-term functional activity changes in elderly and demented patients, and may form the basis for future molecular research into the degree of reliability of neuronal activity and/or cerebral blood flow in abnormal ageing.

\section{Conclusion}

Resting-state fMRI is a reliable tool to measure functional connectivity in the posterior portion of the DMN over a short-term interval. This was supported by the converging results obtained with multiple methodologies investigating network topography and strength. High reliability of the posterior DMN in MCI and AD dementia was confirmed by group comparisons investigating topography and by correlational and consistency models investigating strength. Strength of voxel-based correlation, however, was reduced compared to healthy adults. Activity in the anterior DMN and VPN, on the other hand, showed relatively low signal stability, as indicated by poor statistical reliability. The take home message is that, despite fluctuations in signal connectivity, the posterior DMN is a reliable construct. This bodes well in the potential future translation of posterior DMN indices into potential clinical biomarker proxies.

Acknowledgements This study was partially supported by Grant no. 42/RF-2010-2321718 by the Italian Ministry of Health, and partial funding from the European Union Seventh Framework Program (FP7/2007-2013) under Grant agreement no. 601055, VPH-DARE@ IT to AV. This is a summary of independent research carried out at the NIHR Sheffield Biomedical Research Centre (Translational Neuroscience). The views expressed are those of the author(s) and not necessarily those of the NHS, the NIHR or the Department of Health. We would like to thank Laura Wright for her careful linguistic revision of the manuscript.

Author contributions MCP analysis and interpretation of data and drafting of manuscript. MDM acquisition of data; analysis and interpretation of data; and critical revision. EC analysis and interpretation of data. AV study conception and design; acquisition of data; and critical revision.

\section{Compliance with ethical standards}

Conflict of interest The authors declare that they have no conflict of interest.

Ethical approval All procedures performed in studies involving human participants were in accordance with the ethical standards of the institutional and/or national research committee and with the 1964 Helsinki declaration and its later amendments or comparable ethical standards.

Informed consent Informed consent was obtained from all individual participants included in the study.

OpenAccess This article is distributed under the terms of the Creative Commons Attribution 4.0 International License (http://creativeco mmons.org/licenses/by/4.0/), which permits unrestricted use, distribution, and reproduction in any medium, provided you give appropriate credit to the original author(s) and the source, provide a link to the Creative Commons license, and indicate if changes were made.

\section{References}

1. Chen B, Xu T, Zhou C, Wang L, Yang N, Wang Z, Dong HM, Yang Z, Zang YF, Zuo XN, Weng XC (2015) Individual variability and test-retest reliability revealed by ten repeated resting-state brain scans over one month. PLoS One 10:e0144963

2. Ferreira LK, Busatto GF (2013) Resting-state functional connectivity in normal brain aging. Neurosci Biobehav Rev 37:384-400 
3. Prvulovic D, Bokde AL, Faltraco F, Hampel H (2011) Functional magnetic resonance imaging as a dynamic candidate biomarker for Alzheimer's disease. Prog Neurobiol 95:557-569

4. Sperling R (2011) The potential of functional MRI as a biomarker in early Alzheimer's disease. Neurobiol Aging 32:S37-S43

5. Beltrachini L, De Marco M, Taylor ZA, Lötjönen J, Frangi AF, Venneri A (2015) Integration of cognitive tests and resting state fMRI for the individual identification of mild cognitive impairment. Curr Alzheimer Res 12:592-603

6. Petrella JR, Sheldon FC, Prince SE, Calhoun VD, Doraiswamy PM (2011) Default mode network connectivity in stable vs progressive mild cognitive impairment. Neurology 76:511-517

7. Schouten TM, Koini M, de Vos F, Seiler S, van der Grond J, Lechner A, Hafkemeijer A, Möller C, Schmidt R, de Rooij M, Rombouts SA (2016) Combining anatomical, diffusion, and resting state functional magnetic resonance imaging for individual classification of mild and moderate Alzheimer's disease. Neuroimage Clin 11:46-51

8. Biswal B, Yetkin FZ, Haughton VM, Hyde JS (1995) Functional connectivity in the motor cortex of resting human brain using echo-planar MRI. Magn Reson Med 34:537-541

9. De Luca M, Beckmann CF, De Stefano N, Matthews PM, Smith SM (2006) fMRI resting state networks define distinct modes of long-distance interactions in the human brain. Neuroimage 29:1359-1367

10. Lindquist MA (2008) The statistical analysis of fMRi data. Stat Sci 23:439-464

11. Raichle ME, Mintun MA (2006) Brain work and brain imaging. Annu Rev Neurosci 29:449-476

12. Raichle ME (2015) The restless brain: how intrinsic activity organizes brain function. Philos Trans R Soc Lond B Biol Sci 370:20140172

13. Murphy K, Birn RM, Bandettini PA (2013) Resting-state fMRI confounds and cleanup. Neuroimage 80:349-359

14. Fox MD, Raichle ME (2007) Spontaneous fluctuations in brain activity observed with functional magnetic resonance imaging. Nat Rev Neurosci 8:700-711

15. Seeley WW, Crawford RK, Zhou J, Miller BL, Greicius MD (2009) Neurodegenerative diseases target large-scale human brain networks. Neuron 62:42-52

16. Joo SH, Lim HK, Lee CU (2016) Three large-scale functional brain networks from resting-state functional MRI in subjects with different levels of cognitive impairment. Psychiatry Investig 13:1

17. Lee MH, Smyser CD, Shimony JS (2013) Resting-state fMRI: a review of methods and clinical applications. AJNR Am J Neuroradiol 34:1866-1872

18. Daselaar SM, Prince SE, Cabeza R (2004) When less means more: deactivations during encoding that predict subsequent memory. Neuroimage 23:921-927

19. Buckner RL, Andrews-Hanna JR, Schacter DL (2008) The brain's default network: anatomy, function, and relevance to disease. Ann N Y Acad Sci 1124:1-38

20. Philippi CL, Tranel D, Duff M, Rudrauf D (2015) Damage to the default mode network disrupts autobiographical memory retrieval. Soc Cogn Affect Neurosci 10:318-326

21. Mason MF, Norton MI, Van Horn JD, Wegner DM, Grafton ST, Macrae CN (2007) Wandering minds: the default network and stimulus-independent thought. Science 315:393-395

22. Greicius MD, Srivastava G, Reiss AL, Menon V (2004) Defaultmode network activity distinguishes Alzheimer's disease from healthy aging: evidence from functional MRI. Proc Natl Acad Sci U S A 101:4637-4642

23. Jockwitz C, Caspers S, Lux S, Jütten K, Schleicher A, Eickhoff SB, Amunts K, Zilles K (2017) Age- and function-related regional changes in cortical folding of the default mode network in older adults. Brain Struct Funct 222:83-99

24. Raichle ME, MacLeod AM, Snyder AZ, Powers WJ, Gusnard DA, Shulman GL (2001) A default mode of brain function. Proc Natl Acad Sci U S A 98:676-682

25. Greicius MD, Krasnow B, Reiss AL, Menon V (2003) Functional connectivity in the resting brain: a network analysis of the default mode hypothesis. Proc Natl Acad Sci U S A 100:253-258

26. Jones DT, Machulda MM, Vemuri P, McDade EM, Zeng G, Senjem ML, Gunter JL, Przybelski SA, Avula RT, Knopman DS, Boeve BF, Petersen RC, Jack CR Jr (2011) Age-related changes in the default mode network are more advanced in Alzheimer disease. Neurology 77:1524-1531

27. Davis SW, Dennis NA, Daselaar SM, Fleck MS, Cabeza R (2008) Que PASA? The posterior-anterior shift in aging. Cereb Cortex 18:1201-1209

28. Andrews-Hanna JR, Snyder AZ, Vincent JL, Lustig C, Head D, Raichle ME, Buckner RL (2007) Disruption of large-scale brain systems in advanced aging. Neuron 56:924-935

29. Masters CL, Bateman R, Blennow K, Rowe CC, Sperling RA, Cummings JL (2015) Alzheimer's disease. Nat Rev Dis Primers $1: 15056$

30. Buckner RL, Snyder AZ, Shannon BJ, LaRossa G, Sachs R, Fotenos AF, Sheline YI, Klunk WE, Mathis CA, Morris JC, Mintun MA (2005) Molecular, structural, and functional characterization of Alzheimer's disease: evidence for a relationship between default activity, amyloid, and memory. J Neurosci 25:7709-7717

31. Damoiseaux JS (2012) Resting-state fMRI as a biomarker for Alzheimer's disease? Alzheimers Res Ther 4:8

32. Birn RM, Molloy EK, Patriat R, Parker T, Meier TB, Kirk GR, Nair VA, Meyerand ME, Prabhakaran V (2013) The effect of scan length on the reliability of resting-state fMRI connectivity estimates. Neuroimage 83:550-558

33. Chou YH, Panych LP, Dickey CC, Petrella JR, Chen NK (2012) Investigation of long-term reproducibility of intrinsic connectivity network mapping: a resting-state fMRI study. AJNR Am J Neuroradiol 33:833-838

34. Meindl T, Teipel S, Elmouden R, Mueller S, Koch W, Dietrich O, Coates U, Reiser M, Glaser C (2010) Test-retest reproducibility of the default-mode network in healthy individuals. Hum Brain Mapp 31:237-246

35. Patriat R, Molloy EK, Meier TB, Kirk GR, Nair VA, Meyerand ME, Prabhakaran V, Birn RM (2013) The effect of resting condition on resting-state fMRI reliability and consistency: a comparison between resting with eyes open, closed, and fixated. Neuroimage 78:463-473

36. Shah LM, Cramer JA, Ferguson MA, Birn RM, Anderson JS (2016) Reliability and reproducibility of individual differences in functional connectivity acquired during task and resting state. Brain Behav 6:e00456

37. Shehzad Z, Kelly AM, Reiss PT, Gee DG, Gotimer K, Uddin LQ, Lee SH, Margulies DS, Roy AK, Biswal BB, Petkova E, Castellanos FX, Milham MP (2009) The resting brain: unconstrained yet reliable. Cereb Cortex 19:2209-2229

38. Song J, Desphande AS, Meier TB, Tudorascu DL, Vergun S, Nair VA, Biswal BB, Meyerand ME, Birn RM, Bellec P, Prabhakaran V (2012) Age-related differences in test-retest reliability in resting-state brain functional connectivity. PLoS One 7:e49847

39. Zuo XN, Kelly C, Adelstein JS, Klein DF, Castellanos FX, Milham MP (2010) Reliable intrinsic connectivity networks: testretest evaluation using ICA and dual regression approach. Neuroimage 49:2163-2177

40. Orban P, Madjar C, Savard M, Dansereau C, Tam A, Das S, Evans AC, Rosa-Neto P, Breitner JC, Bellec P; PREVENT-AD Research Group (2015) Test-retest resting-state fMRI in healthy elderly 
persons with a family history of Alzheimer's disease. Sci Data 2:150043

41. Blautzik J, Keeser D, Berman A, Paolini M, Kirsch V, Mueller S, Coates U, Reiser M, Teipel SJ, Meindl T (2013) Long-term test-retest reliability of resting-state networks in healthy elderly subjects and with amnestic mild cognitive impairment patients. $\mathrm{J}$ Alzheimers Dis 34:741-754

42. Nickerson LD, Smith SM, Öngür D, Beckmann CF (2017) Using dual regression to investigate network shape and amplitude in functional connectivity analyses. Front Neurosci 11:115

43. De Marco M, Meneghello F, Duzzi D, Rigon J, Pilosio C, Venneri A (2016) Cognitive stimulation of the default-mode network modulates functional connectivity in healthy aging. Brain Res Bull 121:26-41

44. Sladky R, Friston KJ, Tröstl J, Cunnington R, Moser E, Windischberger C (2011) Slice-timing effects and their correction in functional MRI. Neuroimage 58:588-594

45. Friston KJ, Williams S, Howard R, Frackowiak RS, Turner R (1996) Movement-related effects in fMRI time-series. Magn Reson Med 35:346-355

46. de Flores R, Mutlu J, Bejanin A, Gonneaud J, Landeau B, Tomadesso C, Mézenge F, de La Sayette V, Eustache F, Chételat G (2017) Intrinsic connectivity of hippocampal subfields in normal elderly and mild cognitive impairment patients. Hum Brain Mapp 38:4922-4932

47. Doornweerd S, van Duinkerken E, de Geus EJ, Arbab-Zadeh P, Veltman DJ, Ijzerman RG (2017) Overweight is associated with lower resting state functional connectivity in females after eliminating genetic effects: a twin study. Hum Brain Mapp 38:5069-5081

48. Icenhour A, Witt ST, Elsenbruch S, Lowén M, Engström M, Tillisch K, Mayer EA, Walter S (2017) Brain functional connectivity is associated with visceral sensitivity in women with Irritable Bowel Syndrome. Neuroimage Clin 15:449-457

49. Lazarov A, Zhu X, Suarez-Jimenez B, Rutherford BR, Neria Y (2017) Resting-state functional connectivity of anterior and posterior hippocampus in posttraumatic stress disorder. J Psychiatr Res 94:15-22

50. Onoda K, Yada N, Ozasa K, Hara S, Yamamoto Y, Kitagaki H, Yamaguchi S (2017) Can a resting-state functional connectivity index identify patients with Alzheimer's disease and mild cognitive impairment across multiple sites? Brain Connect 7:391-400

51. D'Agostino E, Maes F, Vandermeulen D, Suetens P (2004) Nonrigid atlas-to-image registration by minimization of class-conditional image entropy. In: Barillot C, Haynor DR, Hellier P (eds) Medical image computing and computer-assisted intervention MICCAI 2004. MICCAI 2004. Lecture notes in computer science, vol. 3216. Springer, Berlin, Heidelberg

52. Friston KJ, Josephs O, Zarahn E, Holmes AP, Rouquette S, Poline J (2000) To smooth or not to smooth? Bias and efficiency in fMRI time-series analysis. Neuroimage 12:196-208

53. Tzourio-Mazoyer N, Landeau B, Papathanassiou D, Crivello F, Etard O, Delcroix N, Mazoyer B, Joliot M (2002) Automated anatomical labeling of activations in SPM using a macroscopic anatomical parcellation of the MNI MRI single-subject brain. Neuroimage 15:273-289

54. Maldjian JA, Laurienti PJ, Kraft RA, Burdette JH (2003) An automated method for neuroanatomic and cytoarchitectonic atlasbased interrogation of fMRI data sets. Neuroimage 19:1233-1239

55. Brett M, Anton JL, Valabregue R, Poline JB (2002) Region of interest analysis using an SPM toolbox. In: Neuroimage 16, 8th international conference on functional mapping of the human brain, Sendai, Japan, 2-6 June 2002

56. Power JD, Mitra A, Laumann TO, Snyder AZ, Schlaggar BL, Petersen SE (2014) Methods to detect, characterize, and remove motion artifact in resting state fMRI. Neuroimage 84:320-341
57. Takeuchi H, Taki Y, Nouchi R, Yokoyama R, Kotozaki Y, Nakagawa S, Sekiguchi A, Iizuka K, Yamamoto Y, Hanawa S, Araki T, Makoto Miyauchi C, Shinada T, Sakaki K, Nozawa T, Ikeda S, Yokota S, Daniele M, Sassa Y, Kawashima R (2017) Regional homogeneity, resting-state functional connectivity and amplitude of low frequency fluctuation associated with creativity measured by divergent thinking in a sex-specific manner. Neuroimage 152:258-269

58. Murphy K, Birn RM, Handwerker DA, Jones TB, Bandettini PA (2009) The impact of global signal regression on resting state correlations: are anti-correlated networks introduced? Neuroimage 44:893-905

59. De Marco M, Duzzi D, Meneghello F, Venneri A (2017) Cognitive efficiency in Alzheimer's disease is associated with increased occipital connectivity. J Alzheimers Dis 57:541-556

60. Kalcher K, Huf W, Boubela RN, Filzmoser P, Pezawas L, Biswal B, Kasper S, Moser E, Windischberger C (2012) Fully exploratory network independent component analysis of the 1000 functional connectomes database. Front Hum Neurosci 6:301

61. Franco AR, Pritchard A, Calhoun VD, Mayer AR (2009) Interrater and intermethod reliability of default mode network selection. Hum Brain Mapp 30:2293-2303

62. Shaffer JP (1995) Multiple hypothesis testing. Annu Rev Psychol 46:561-584

63. Koo TK, Li MY (2016) A guideline of selecting and reporting intraclass correlation coefficients for reliability research. J Chiropr Med 15:155-163

64. Bland JM, Altman DG (1997) Cronbach's alpha. BMJ 314:572

65. Casanova R, Srikanth R, Baer A, Laurienti PJ, Burdette JH, Hayasaka S, Flowers L, Wood F, Maldjian JA (2007) Biological parametric mapping: a statistical toolbox for multimodality brain image analysis. Neuroimage 34:137-143

66. Power JD, Barnes KA, Snyder AZ, Schlaggar BL, Petersen SE (2012) Spurious but systematic correlations in functional connectivity MRI networks arise from subject motion. Neuroimage 59:2142-2154

67. Zuo XN, Xing XX (2014) Test-retest reliabilities of resting-state FMRI measurements in human brain functional connectomics: a systems neuroscience perspective. Neurosci Biobehav Rev 45:100-118

68. Shoukri MM, Elkum N, Walter SD (2006) Interval estimation and optimal design for the within-subject coefficient of variation for continuous and binary variables. BMC Med Res Methodol 6:24

69. Zandbelt BB, Gladwin TE, Raemaekers M, van Buuren M, Neggers SF, Kahn RS, Ramsey NF, Vink M (2008) Within-subject variation in BOLD-fMRI signal changes across repeated measurements: quantification and implications for sample size. Neuroimage 42:196-206

70. Müller R, Büttner P (1994) A critical discussion of intraclass correlation coefficients. Stat Med 13:2465-2476

71. Chang C, Glover GH (2009) Effects of model-based physiological noise correction on default mode network anti-correlations and correlations. Neuroimage 47:1448-1459

72. Hutton C, Josephs O, Stadler J, Featherstone E, Reid A, Speck O, Bernarding J, Weiskopf N (2011) The impact of physiological noise correction on fMRI at $7 \mathrm{~T}$. Neuroimage 57:101-112

73. Pruim RHR, Mennes M, van Rooij D, Llera A, Buitelaar JK, Beckmann CF (2015) ICA-AROMA: a robust ICA-based strategy for removing motion artifacts from fMRI data. Neuroimage 112:267-277

74. Power JD, Schlaggar BL, Petersen SE (2015) Recent progress and outstanding issues in motion correction in resting state fMRI. Neuroimage 105:536-551

75. Goto M, Abe O, Miyati T, Yamasue H, Gomi T, Takeda T (2016) Head motion and correction methods in resting-state functional MRI. Magn Reson Med Sci 15:178-186 
76. McAvoy M, Larson-Prior L, Nolan TS, Vaishnavi SN, Raichle ME, d'Avossa G (2008) Resting states affect spontaneous BOLD oscillations in sensory and paralimbic cortex. J Neurophysiol 100:922-931

77. Damoiseaux JS, Rombouts SA, Barkhof F, Scheltens P, Stam CJ, Smith SM, Beckmann CF (2006) Consistent resting-state networks across healthy subjects. Proc Natl Acad Sci U S A 103:13848-13853

78. Raichle ME (2015) The brain's default mode network. Annu Rev Neurosci 38:433-447

79. Eklund A, Nichols TE, Knutsson H (2016) Cluster failure: why fMRI inferences for spatial extent have inflated false-positive rates. Proc Natl Acad Sci U S A 113:7900-7905

80. Button KS, Ioannidis JP, Mokrysz C, Nosek BA, Flint J, Robinson ES, Munafò MR (2013) Power failure: why small sample size undermines the reliability of neuroscience. Nat Rev Neurosci $14: 365-376$
81. Braak H, Braak E (1991) Neuropathological stageing of Alzheimer-related changes. Acta Neuropathol 82:239-259

82. Rombouts SA, Barkhof F, Goekoop R, Stam CJ, Scheltens P (2005) Altered resting state networks in mild cognitive impairment and mild Alzheimer's disease: an fMRI study. Hum Brain Mapp 26:231-239

83. Stern Y (2009) Cognitive reserve. Neuropsychologia 47:2015-2028

Publisher's Note Springer Nature remains neutral with regard to jurisdictional claims in published maps and institutional affiliations. 